PROCEEDINGS OF THE

AMERICAN MATHEMATICAL SOCIETY

Volume 27, Number 3, March 1971

\title{
ON A PARTITION THEOREM OF MACMAHON-ANDREWS
}

\author{
M. V. SUBBARAO
}

Abstract. Two theorems are given about partitions in which the multiplicity of the parts satisfies certain conditions. One of these theorems generalizes a recent result of Andrews concerning partitions in which a part with an odd multiplicity occurs at least $2 r+1$ times.

Recently, George Andrews [1] proved the following partition theorem, generalizing an earlier result of MacMahon [2, p. 54] (which deals with the case $r=1$ ):

The number of partitions of $n$, in which a part occurring an odd number of times occurs at least $(2 r+1)$ times, equals the number of partitions of $n$ into parts which are either even or else $\equiv 2 r+1$ $(\bmod 4 r+2)$.

We wish to remark that Andrews' theorem is itself a special case of the following result.

THEOREM (A). Let $k$ be any integer $>1$ and $l$ any positive integer $\not \equiv 0(\bmod k)$. Let $A_{k, l}(n)$ be the number partitions of $n$ in which the multiplicity of each part is either $\equiv 0(\bmod k)$ or else $\geqq l$ and $\equiv l(\bmod$ $k)$. Let $B_{k, l}(n)$ denote the number of partitions of $n$ in which the parts are either $\equiv 0(\bmod k)$ or else $\equiv l(\bmod 2 l)$. Then $A_{k, l}(n)=B_{k, l}(n)$.

Andrews' result corresponds to the choice $k=2, l=2 r+1$. The proof of this is analogous to that of Andrews' and is therefore omitted.

It is possible to obtain several results of this kind. As a sample, we give the following:

THEOREM B. Let $m>1, r \geqq 0$ be integers, and let $C_{m, r}(n)$ be the number of partitions of $n$ such that all even multiplicities of the parts are less than $2 m$, and all odd multiplicities are at least $2 r+1$ and at most $2(m+r)-1$. Let $D_{m, r}(n)$ be the number of partitions of $n$ into parts which are either odd and $\equiv 2 r+1(\bmod 4 r+2)$, or even and $\neq 0$ $(\bmod 2 m)$. Then $C_{m, r}(n)=D_{m, r}(n)$.

Received by the editors May 25, 1970.

AMS 1969 subject classifications. Primary 1055.

Key words and phrases. Partitions, multiplicity of a part, a formula of Euler.

Copyright (c) 1971, American Mathematical Society 
Proof.

$1+\sum_{n=1}^{\infty} C_{m, r}(n) x^{n}=\prod_{n=1}^{\infty}\left\{1+x^{2 n}+x^{4 n}+\cdots+x^{(2 m-2) n}+x^{(2 r+1) n}\right.$

$$
\begin{aligned}
& \left.\quad+x^{(2 r+3) n}+\cdots+x^{(2 m+2 r-1) n}\right\} \\
& =\prod_{n=1}^{\infty}\left(1-x^{2 m n}\right)\left(1-x^{2 n}\right)^{-1}\left(1+x^{(2 r+1) n}\right) \\
& =\prod_{n=1 ; k \neq 0(\bmod m)}^{\infty}\left(1-x^{2 k n}\right)^{-1} \prod_{n=1}^{\infty}\left(1-x^{(2 n-1)(2 r+1)}\right)^{-1} \\
& =1+\sum_{n=1}^{\infty} D_{m, r}(n) x^{n},
\end{aligned}
$$

where we used a well-known Euler identity [2, pp. 10-11], to transform the last product in (1) into the last product in (2). This completes the proof.

The above two theorems can of course be restated using, for the definitions of $A_{k, l}(n), B_{k, l}(n), C_{m, r}(n)$ and $D_{m, r}(n)$, the conjugates of the concerned partitions.

As a particularly interesting special case of the last theorem, we obtain, on taking $m=2, r=1$, the following:

COROLLARY. The number of partitions of $n$, in which each part occurs two, three or five times, equals the number of partitions of $n$ into parts which are of the forms $2(\bmod 4)$ or $3(\bmod 6)$.

\section{REFERENCES}

1. George E. Andrews, $A$ generalization of a partition theorem of MacMahon, J. Combinatorial Theory 3 (1967), 100-101. MR 35 \#2766.

2. P. A. MacMahon, Combinatory analysis, Vol. 2, Reprint Chelsea, New York, 1960. MR $25 \# 5003$.

University of Alberta, Edmonton, Alberta, Canada 